\title{
Three Epochs of Star Formation in the High\# Redshift Universe
}

\section{Citation}

Mackey, Jonathan, Volker Bromm, and Lars Hernquist. 2003. "Three Epochs of Star Formation in the High\#Redshift Universe." The Astrophysical Journal 586 (1): 1-11. https:// doi.org/10.1086/367613.

\section{Permanent link}

http://nrs.harvard.edu/urn-3:HUL.InstRepos:41381647

\section{Terms of Use}

This article was downloaded from Harvard University's DASH repository, and is made available under the terms and conditions applicable to Other Posted Material, as set forth at http:// nrs.harvard.edu/urn-3:HUL.InstRepos:dash.current.terms-of-use\#LAA

\section{Share Your Story}

The Harvard community has made this article openly available.

Please share how this access benefits you. Submit a story.

Accessibility 


\title{
Three Epochs of Star Formation in the High Redshift Universe
}

\author{
Jonathan Mackey, Volker Bromm, and Lars Hernquist \\ Harvard-Smithsonian Center for Astrophysics, 60 Garden Street, Cambridge, MA 02138; \\ jmackey@cfa.harvard.edu,vbromm@cfa.harvard.edu,lars@cfa.harvard.edu
}

\begin{abstract}
We investigate the impact of an early population of massive stars on their surroundings. Dissociation of molecular hydrogen by strong UV emission from such stars is expected to produce a global transition in the cooling mechanism of minihalos at a redshift of approximately 30, strongly inhibiting star formation until more massive halos can collapse. Furthermore, chemical enrichment from Pop III supernovae produces a second transition at $z \sim 15-20$, when the mean metallicity of the universe exceeds a critical threshold and Pop III star formation gives way to Pop II. We show that observations of high redshift supernovae with the Next Generation Space Telescope (NGST) have the potential to trace the cosmic star formation rate out to $z \gtrsim 20$, provided that Pop III supernovae are at least as bright as, and ideally brighter than, type Ia supernovae. We also propose a mechanism for the formation of a novel population of extremely low metallicity stars of intermediate mass at very high redshifts, which we term Pop II.5. In our model shock compression, heating, and subsequent cooling to high density reduces the fragment mass in primordial gas to $\sim 10 \mathrm{M}_{\odot}$, allowing low mass stars to form. We predict the number density of relic Pop II.5 stars in the Milky Way halo today and find that, with certain assumptions, there should be $\sim 10 \mathrm{kpc}^{-3}$ in the solar neighborhood.
\end{abstract}

Subject headings: cosmology: theory — early universe — galaxies: formation — intergalactic medium — stars: formation

\section{Introduction}

An important challenge in modern cosmology is to understand how the cosmic "dark ages" ended (for a recent review, see Barkana \& Loeb 2001). There is growing theoretical evidence indicating that the first luminous objects to form in the universe were very massive stars with typical masses $M_{*} \gtrsim 100 \mathrm{M}_{\odot}$ (Bromm, Coppi \& Larson 1999, 2002; Abel, Bryan \& 
Norman 2000, 2002; Nakamura \& Umemura 2001). These stars formed out of metal-free gas in dark matter $(\mathrm{DM})$ halos of mass $\sim 10^{5}-10^{6} \mathrm{M}_{\odot}$ at redshifts $z \gtrsim 20$ (e.g., Tegmark et al. 1997; Fuller \& Couchman 2000). Typically, simulations predict that the halos hosting this process should contain either one or a few dense massive clumps of baryonic matter, with the most massive clump situated nearest to the center of the halo being the one to collapse first and to presumably form a very massive star (VMS). On the other hand, conditions in the universe less than $10^{9} \mathrm{yr}$ after the big bang must have allowed for the formation of low-mass stars, with typical masses of $\sim 1 \mathrm{M}_{\odot}$, as is implied by the ages of the oldest globular clusters in the Galactic halo with metallicities of $Z \sim 10^{-2} \mathrm{Z}_{\odot}$ (e.g., Ashman \& Zepf 1998; Bromm \& Clarke 2002). In this paper, we investigate the question: How and when did the transition from the formation of very massive stars at high redshifts to that of more normal, low-mass stars at later times take place?

Observations have recently provided hints to the character of star formation at $z \gtrsim 5$. The abundance of C IV in the low-column density Ly $\alpha$ forest indicates that the intergalactic medium (IGM) was enriched with heavy elements to a level of $Z \sim 10^{-3.5} \mathrm{Z}_{\odot}$ already at $z \sim 5$ (Songaila 2001). In addition, abundance patterns in Ly $\alpha$ systems out to $z \sim 4.6$ are suggestive of an early, prompt nucleosynthetic inventory of heavy elements from a generation of very massive stars (Qian, Sargent \& Wasserburg 2002).

Elucidating the cosmic star formation history in the high redshift universe is crucial for predicting what observatories such as the Next Generation Space Telescope (NGST) will observe at $z>5$ less than a decade from now. In particular, it is important to ascertain the rates and properties of high redshift supernovae ( $\mathrm{SNe}$ ) which are likely to be the brightest beacons heralding the end of the "dark ages" (Miralda-Escudé \& Rees 1997). Similarly, gamma-ray bursts (GRBs) are expected to trace the formation history of massive stars out to very high redshifts (Totani 1997; Wijers et al. 1998; Blain \& Natarajan 2000). In fact, the top-heavy initial mass function (IMF) predicted for the first stars favors the massive stars which are the likely source of GRB progenitors. Constraining how stars form at high $z$, therefore, is of great relevance for interpreting the results of the upcoming Swift satellite (Bromm \& Loeb 2002).

Here, we examine processes related to star formation in the high redshift universe using a simple analytical model that synthesizes a number of recent theoretical and observational results into a coherent framework. This idealized model is intended to emphasize the basic physical mechanisms, and we will complement it with detailed numerical simulations in future work.

The organization of the paper is as follows. In $\S 2$ we describe our high-redshift star formation model. A discussion of the various stellar populations that form at high $z$ is given 
in $\S 3$, while $\S 4$ presents observational consequences. Finally, $\S 5$ contains our conclusions and the outlook for future work.

\section{Epochs of Star Formation at High Redshift}

\subsection{Cosmic Star Formation History}

In general, VMSs will impart three forms of feedback on the IGM (e.g., Ciardi et al. 2000b): radiative, chemical, and mechanical. Owing to their high effective temperatures, $T_{\text {eff }} \sim 10^{5} \mathrm{~K}$, these stars produce a large quantity of UV photons, roughly 10 times as many per stellar baryon as those from a "normal" stellar population characterized by a Salpeter IMF (Tumlinson \& Shull 2000; Bromm, Kudritzki \& Loeb 2001b). Upon its death after only $\sim 3 \times 10^{6} \mathrm{yr}$, a VMS explodes as a supernova and enriches the IGM with heavy elements, provided the star is not more massive than $\sim 260 \mathrm{M}_{\odot}$, in which case it is predicted to collapse entirely into a massive black hole without any concomitant metal ejection (Fryer, Woosley \& Heger 2001). We propose that this radiative and chemical feedback gives rise to two fundamental transitions in the cosmic star formation history at high $z$. The feedback from mechanical energy input into the IGM might lead to a distinct new population of stars, to be discussed in $\S 3$.

To assess the global feedback exerted by VMSs on the IGM, we need to know their formation rate. We calculate this rate according to the "single-burst" model of VMS formation introduced by Santos, Bromm \& Kamionkowski (2002). This model adopts the observationally favored scenario in which the formation of cosmic structure has progressed hierarchically from small to large scales as described by the cold dark matter (CDM) model with a cosmological constant. Specifically, we assume a $\Lambda$ CDM model with density parameters in matter $\Omega_{m}=1-\Omega_{\Lambda}=0.3$ and in baryons $\Omega_{B}=0.045$, a Hubble parameter of $h=H_{0} /\left(100 \mathrm{~km} \mathrm{~s}^{-1} \mathrm{Mpc}^{-1}\right)=0.7$, and a scale-invariant power spectrum of density fluctuations with a normalization $\sigma_{8}=0.9$. The abundance and merger history of the DM halos is given by the extended Press-Schechter formalism (Lacey \& Cole 1993). Within merging halos, stars form in gas that is able to cool efficiently, via either molecular or atomic hydrogen lines. These cooling mechanisms become effective at different minimum temperatures, $T_{\text {crit }} \sim 400 \mathrm{~K}$ and $\sim 10^{4} \mathrm{~K}$ for molecular and atomic hydrogen, respectively (e.g., Barkana \& Loeb 2001). These temperature thresholds correspond to redshift-dependent minimum DM halo masses

$$
M_{\text {crit }}(z) \simeq 10^{8} \mathrm{M}_{\odot}\left(\frac{\mu}{0.6}\right)^{-3 / 2}\left(\frac{T_{\text {crit }}}{10^{4} \mathrm{~K}}\right)^{3 / 2}\left(\frac{1+z}{10}\right)^{-3 / 2},
$$

where $\mu$ is the mean molecular weight ( $\mu=0.6$ for ionized gas, and $\mu=1.2$ for neutral gas). 


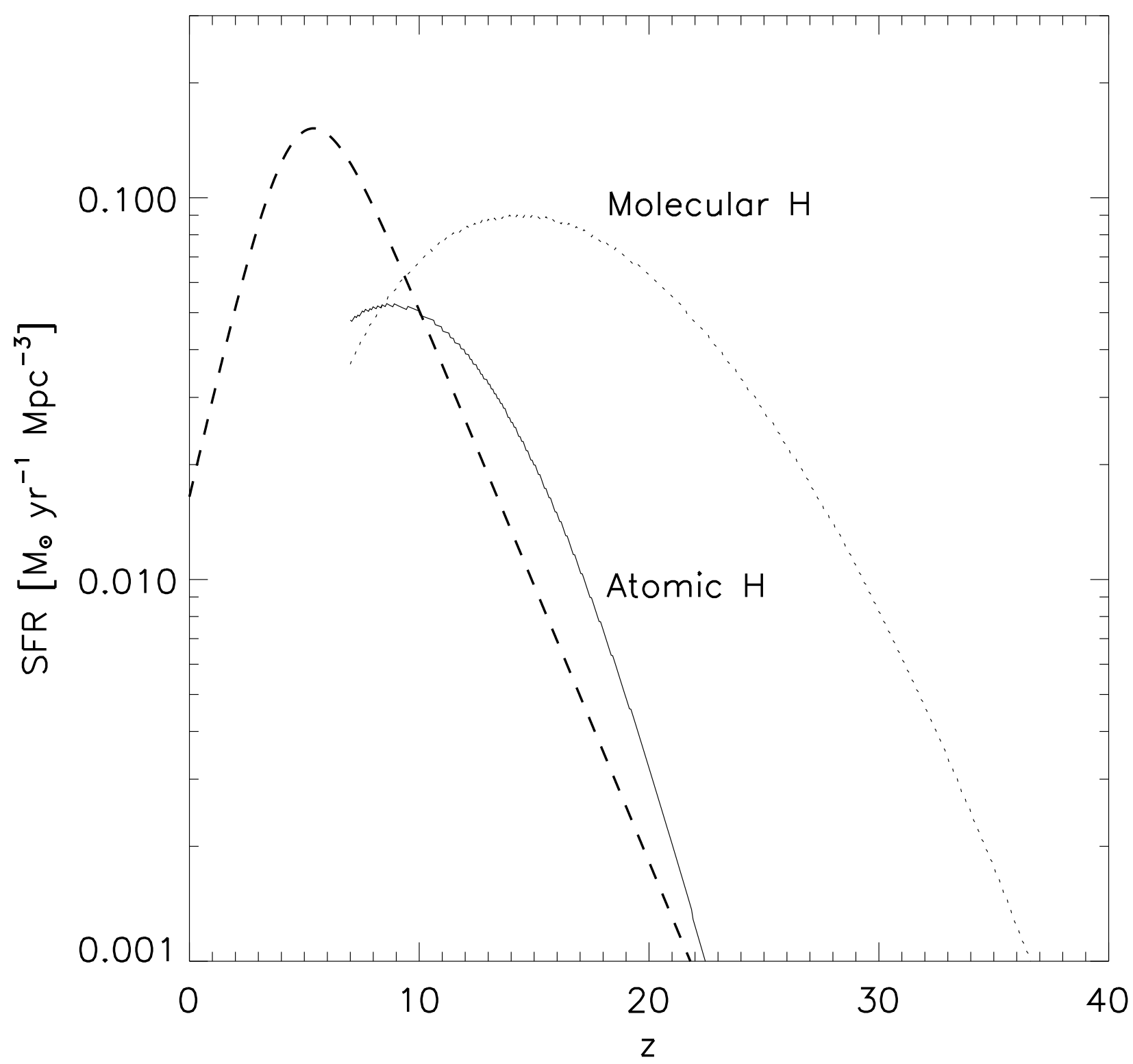

Fig. 1.- History of comoving star formation rate (SFR) density in units of $\mathrm{M}_{\odot} \mathrm{yr}^{-1} \mathrm{Mpc}^{-1}$ as a function of redshift. Solid line: Formation rate of VMSs in halos that cool due to atomic hydrogen. Dotted line: VMS formation rate in halos that cool due to molecular hydrogen. In calculating these rates, VMS formation was assumed to proceed in a single-burst mode with an efficiency of $\eta_{*}=10 \%$ (see Santos et al. 2002). Dashed line: Analytic fit to the star formation history derived from cosmological simulations that incorporate a multi-phase model of quiescent star formation (from Springel \& Hernquist 2002). 
Finally, the star formation efficiency is chosen to be $\eta_{*}=0.10$. The "single-burst" model now assumes that VMS formation is accompanied by strong negative feedback effects on the star-forming gas (see below for a detailed discussion). In essence, VMSs are only allowed to form out of gas that has never perviously experienced star formation, in merging DM halos that cross $M_{\text {crit }}(z)$ for the first time.

We only consider mergers in which the two progenitor halos are within a factor of 100 of each other in mass, although our results are quite similar (within a factor of 2) if this ratio is as low as 10 or as high as 300. We impose this limit because very minor mergers will not set off a burst of VMS formation, and also because they take a long time. Dynamical friction cannot cause halos to merge within a Hubble time if their mass ratio is large. Any uncertainty in the allowed mass ratio is significantly smaller than the uncertainty in the value of $\eta_{*}$.

Figure 1 shows the resulting star formation histories. We here presume that VMS formation will have ceased at latest by $z \sim 7$, close to the estimated redshift of reionization (Barkana 2002, and references therein). The precise redshift where VMS formation ends is uncertain, and is determined by the feedback effects that we will discuss below. For the sake of comparison, in Figure 1 we also show an analytical fit to the star formation history obtained by Springel \& Hernquist (2002) from a series of cosmological simulations that incorporate a self-regulated model of quiescent star formation in a multi-phase interstellar medium. This latter history describes the formation of a normal, predominantly low-mass population of stars (see §3). Note that the Springel \& Hernquist results were obtained for a cosmology with a slightly smaller value of $\Omega_{B}=0.04$ than for our current analysis.

\subsection{Radiative Feedback and the First Transition in the Star Formation History}

In a hierarchical model of structure formation, the very first stars will form in small DM halos where gas cooling has to rely on the presence of molecular hydrogen. Initially, therefore, star formation proceeds along the molecular branch in Figure 1, and is characterized by a top-heavy IMF. This latter prediction follows from the microphysics of $\mathrm{H}_{2}$ cooling, leading to characteristic values of the gas temperature and density, thus imprinting a characteristic mass scale of a few $100 \mathrm{M}_{\odot}$ (Bromm et al. 2002). The very first stars derive from $3-4 \sigma$ peaks and, albeit rare, efficiently produce Lyman-Werner (LW) photons with energies (11.2$13.6 \mathrm{eV}$ ) just below the hydrogen ionization threshold. The LW photons are therefore free to propagate through an otherwise neutral IGM, readily destroying $\mathrm{H}_{2}$ in neighboring halos (Haiman, Rees \& Loeb 1997; Haiman, Abel \& Rees 2000; Ricotti, Gnedin \& Shull 2001; but 
see Ciardi, Ferrara \& Abel 2000a).

Very massive stars produce approximately $\sim 10^{4} \mathrm{LW}$ photons per stellar baryon (Bromm et al. 2001b). Roughly, one can then estimate the redshift where star formation in low-mass halos (relying on $\mathrm{H}_{2}$ cooling) is no longer possible by demanding that there be of order one dissociating LW photon for each molecule in the IGM:

$$
10^{4} f_{\mathrm{LW}} f_{\text {diss }} \rho_{*}\left(z_{1 \rightarrow 2}\right) \sim f_{\mathrm{H}_{2}} \rho_{\mathrm{B}} .
$$

Here, $\rho_{\mathrm{B}}=4.1 \times 10^{-31} \mathrm{~g} \mathrm{~cm}^{-3}$ is the comoving density in baryons, $f_{\mathrm{LW}}$ the escape fraction of LW photons from the star-forming halo, $f_{\text {diss }}$ the fraction of absorptions that lead to dissociation, $f_{\mathrm{H}_{2}}$ the molecule fraction in the IGM, and

$$
\rho_{*}(z)=\int_{z}^{\infty} \psi_{*}\left(z^{\prime}\right)\left|\frac{\mathrm{d} t}{\mathrm{~d} z^{\prime}}\right| \mathrm{d} z^{\prime}
$$

is the comoving density in stars, with $\psi_{*}(z)$ being the comoving star formation rate (SFR), in this case given by the molecular track in Figure 1, and the other symbols have their usual meaning (e.g., Bromm \& Loeb 2002).

In Figure 2, we evaluate this criterion with $f_{\mathrm{LW}}=0.1, f_{\text {diss }}=0.1$ (Glover \& Brand 2001) and $f_{\mathrm{H}_{2}}=10^{-3}$, finding $z_{1 \rightarrow 2} \gtrsim 30$. We interpret this redshift as the approximate boundary between the first two epochs of cosmic star formation. Notice that this is a rather conservative estimate, as the fraction of gas in molecular form is only $\sim 10^{-6}-10^{-3}$, and the required number of LW photons might therefore be significantly smaller than the value derived from equation (2). It is also worth pointing out that such an early transition redshift is a direct consequence of the predicted top-heavy IMF for the first stars, with a factor $\gtrsim 10$ enhancement in the production of LW photons per stellar baryon compared to the case of a standard Salpeter IMF (Bromm et al. 2001b). We have verified that this transition redshift is not very sensitive to variations within factors of a few in $f_{\mathrm{LW}}$ and $f_{\text {diss }}$.

At lower redshifts, star formation can now only proceed in halos that are massive enough to cool via atomic hydrogen lines (corresponding to masses $M \gtrsim 10^{8} \mathrm{M}_{\odot}$ ). To form stars, the gas has to cool below the $\sim 10^{4} \mathrm{~K}$ that can be reached by atomic line cooling. Ultimately, therefore, star formation in these massive halos still relies on cooling due to $\mathrm{H}_{2}$ which is expected to form in dense regions that are shielded from the LW background (Oh \& Haiman 2002). The same physical reasoning that leads to the prediction of a top-heavy IMF in small, $\sim 10^{6} \mathrm{M}_{\odot}$, halos should then also apply to these more massive halos, regardless of whether $\mathrm{H}_{2}$ is initially present in them or not. The formation of stars along the atomic track (see Figure 2) during epoch 2 consequently proceeds with the same top-heavy IMF as in epoch 1. 


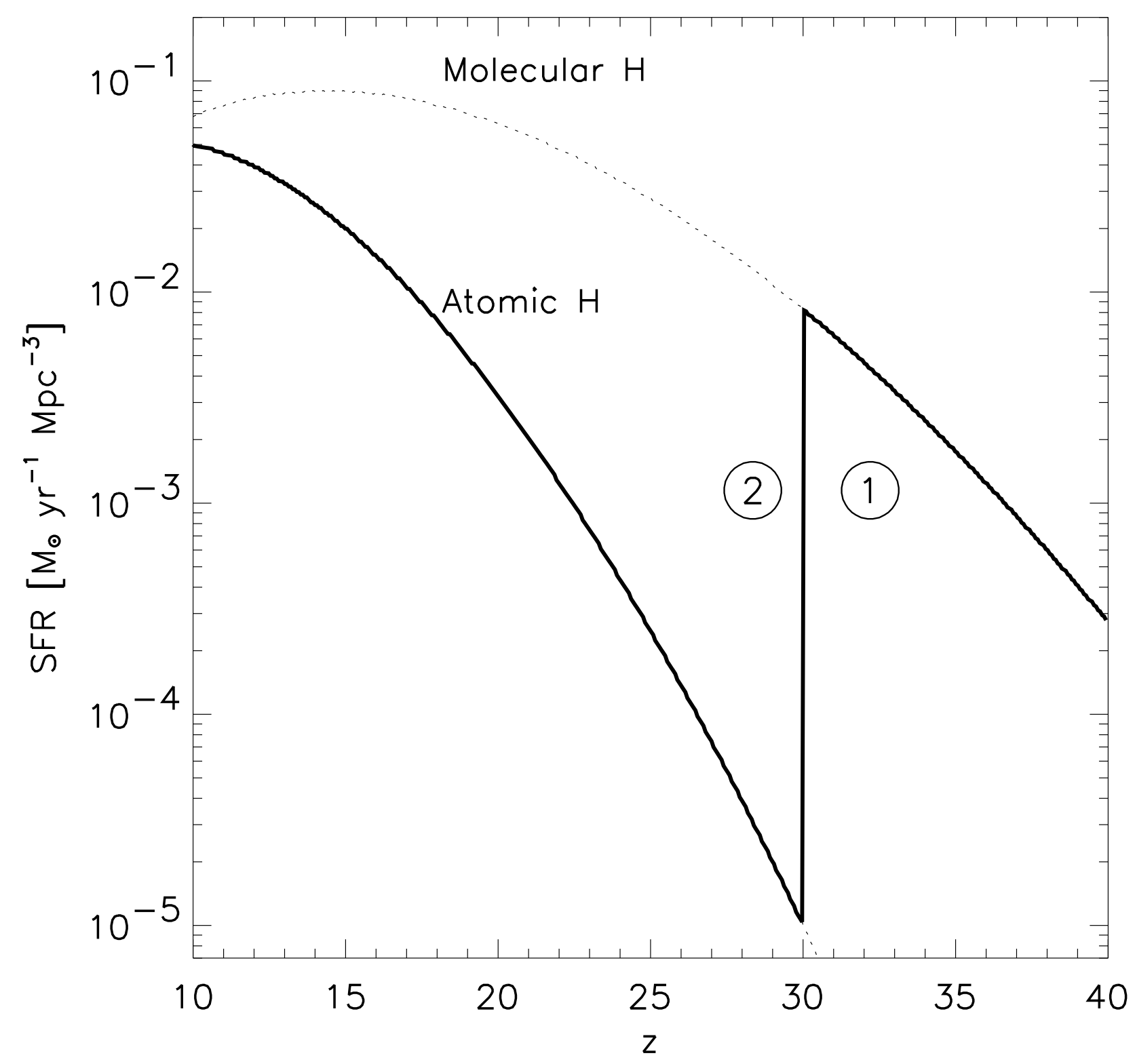

Fig. 2.- The transition from epoch 1 to 2. Comoving star formation rate (SFR) in units of $\mathrm{M}_{\odot} \mathrm{yr}^{-1} \mathrm{Mpc}^{-3}$ as a function of redshift. Dotted lines: VMS formation rate calculated for cooling due to atomic and to molecular hydrogen, respectively (from Figure 1). Solid line: The actually realized star formation history. Once LW-photodissociation feedback becomes effective at $z \sim 30$, the SFR jumps from the molecular to the atomic track. This jump marks the transition from the first to the second epoch of high- $z$ star formation. 
The break in the star formation rate at $z=30$ is artificially sharp due to the simplicity of our modeling. In reality, this is likely to be a smoother transition because the molecular gas will not be dissociated instantaneously, and will not be completely dissociated in more massive halos (Machacek, Bryan \& Abel 2001). We do believe, however, that this transition will be rapid. The mean free path of LW photons at $z=30$ is $\lambda_{\mathrm{mfp}} \sim 10 \mathrm{Mpc}$ (physical), much larger than the typical separation of $10^{6} \mathrm{M}_{\odot}$ halos at this redshift $(\sim 30 \mathrm{kpc})$. Photons travel this mean free path in $\sim 10^{7} \mathrm{yr}$, which is short compared to the Hubble time. For these reasons, a roughly uniform background of LW photons should be set up very quickly. The number of sources increases exponentially with time, so the background radiation level will increase rapidly until it is intense enough to dissociate most of the molecules in the universe.

The timescale for photodissociation of a molecule is inversely proportional to the intensity of the radiation field in the Lyman-Werner bands, $J_{\mathrm{LW}}$, and also depends on how well the molecule is shielded from the radiation. Oh \& Haiman (2002) give the relationship as

$$
t_{\text {diss }}=2.1 \times 10^{4} J_{21}^{-1} f_{\text {shield }}^{-1} \mathrm{yr},
$$

where $J_{21}=J_{\mathrm{LW}} / 10^{-21} \mathrm{erg} \mathrm{cm}^{-2} \mathrm{~s}^{-1} \mathrm{~Hz}^{-1} \mathrm{sr}^{-1}$, and $f_{\text {shield }}$ takes into account the effects of $\mathrm{H}_{2}$ self-shielding and is given by $f_{\text {shield }}=\min \left[1,\left(N_{\mathrm{H}_{2}} / 10^{14} \mathrm{~cm}^{-2}\right)^{-0.75}\right]$ (Draine \& Bertoldi 1996). Thus, the dissociation time at fixed column density depends only on the background radiation intensity, approximately given by

$$
J_{\mathrm{LW}} \simeq \frac{10^{4} h c}{4 \pi m_{\mathrm{H}}} \rho_{*}(z) \exp \left[-\tau_{\mathrm{IGM}}\right],
$$

assuming $10^{4} \mathrm{LW}$ photons are produced per baryon of star formation (see above). The constants in this expression have their usual meaning. The optical depth of the IGM to LW photons is estimated to be $\tau_{\mathrm{IGM}} \simeq 2-3$ (e.g., Ciardi et al. 2000a; Ricotti et al. 2001), reducing the UV flux by about an order of magnitude. Our formula for $J_{\text {LW neglects the }}$ redshifting of photons out of the LW bands, but photons travel a mean free path in a small fraction of the Hubble time and so should be absorbed long before redshift effects become important.

In Figure 3 we show the dissociation time compared to the Hubble time as a function of redshift for different neutral hydrogen column densities. We also show the lifetime of a VMS for comparison. We have calculated the curves in Figure 3 for a molecular fraction of $10^{-3}$, and with $\tau_{\mathrm{IGM}}=2$. A $10^{6} \mathrm{M}_{\odot}$ halo at $z=30$ would have a hydrogen column density of $\sim 4 \times 10^{20} \mathrm{~cm}^{-2}$ if it were a uniform sphere, and about 10 times larger if it had an isothermal density distribution (Glover \& Brand 2001). To relate this to the rarity of the peaks, we again consider $z=30$. A 3-sigma peak has a mass of only $4 \times 10^{3} \mathrm{M}_{\odot}$, and 


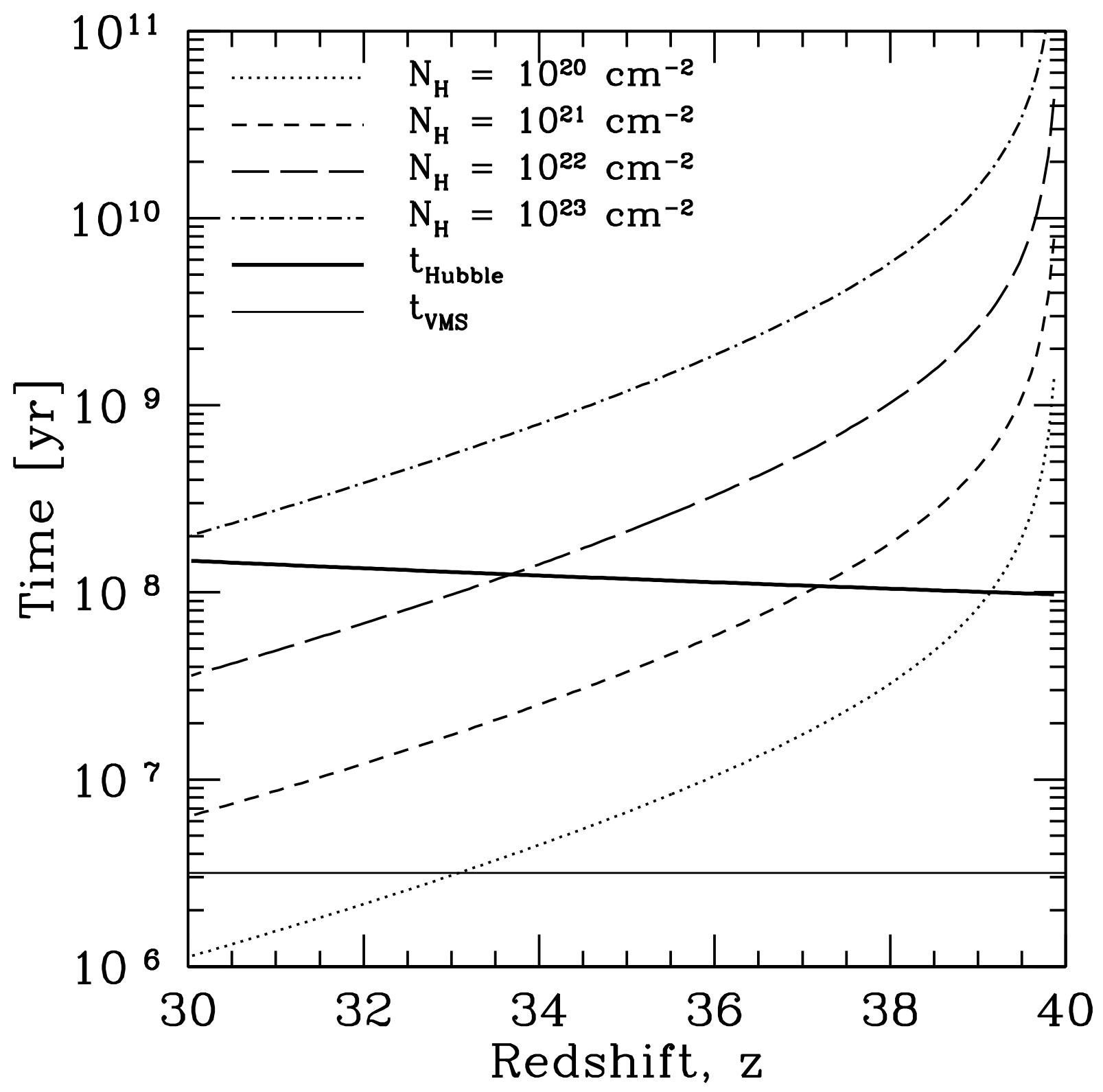

Fig. 3. - The photodissociation time for molecular hydrogen as a function of redshift. The heavy solid line is the Hubble time, the light solid line is the lifetime of a VMS ( $\sim 3 \mathrm{Myr})$, and the four broken lines are the dissociation times in halos with different neutral hydrogen column densities. Dissociation times decrease towards lower redshifts because the soft-UV background radiation is increasing rapidly in intensity. 
so does not collapse. The extremely rare peaks in the density field are thus responsible for dissociating the hydrogen molecules. The masses of halos corresponding to 4-, 5-, and 6-sigma peaks are $\sim 5 \times 10^{5}, 1 \times 10^{7}$, and $1 \times 10^{8} \mathrm{M}_{\odot}$, respectively. For a truncated isothermal sphere, this corresponds to column densities towards the center of the halos of

$N_{\mathrm{H}} \sim 6 \times 10^{21}, 2 \times 10^{22}$, and $4 \times 10^{22} \mathrm{~cm}^{-2}$. Comparing these column densities with the respective curves in Figure 3, we see that the dissociation timescales are always less than the Hubble time for even the rarest objects by $z=30$. Thus we tentatively conclude that this transition is indeed likely to be rapid.

\subsection{Chemical Feedback and the Second Transition in the Star Formation History}

Here, we make the simplifying assumption that all VMSs have masses in the range $140 \lesssim M \lesssim 260 \mathrm{M}_{\odot}$, and that they consequently explode as pair-instability supernovae (PISNe) which completely disrupt the star without leaving behind a compact remnant (Fryer et al. 2001; but see Umeda \& Nomoto 2002). PISNe are predicted to have substantial metal yields, of order $y=M_{Z} / M_{*} \sim 0.50$ (Heger \& Woosley 2002). The heavy elements that are produced in the SN explosions will enrich the host halo, and a fraction, $f_{\text {mix }}$, of them will escape into the general IGM as a result of SN-driven outflows (e.g., Madau, Ferrara \& Rees 2001; Mori, Ferrara \& Madau 2002; Thacker, Scannapieco \& Davis 2002). While in $\S 3$ we predict that some low mass star formation will occur simultaneously with VMS formation, these stars will explode as type II supernovae which have a much lower metal yield $\left(\sim 1 \mathrm{M}_{\odot}\right.$ per supernova). This means that their contribution to the metallicity of the universe is small as long as there is comparable star formation in VMSs, and we thus ignore any contribution from low mass stars in the following calculation.

With the star formation history described in Figure 2, we now calculate the resulting metal enrichment of the IGM

$$
Z_{\mathrm{IGM}}(z)=y f_{\operatorname{mix}} \frac{\rho_{*}(z)}{\rho_{\mathrm{B}}},
$$

where $\rho_{*}(z)$ is the cumulative density in stars as given in equation (3). The crucial uncertainty is the fraction, $f_{\text {mix }}$, of metals that are able to escape the star forming system, and to get mixed into the general IGM. For simplicity, we assume that all the metals escaping a star forming halo are mixed uniformly into the IGM. To bracket the likely cases, we calculate $Z_{\mathrm{IGM}}(z)$ for two values of the mixing efficiency: $f_{\text {mix }}=0.05$ and 0.5 . It appears likely that the presence of metals is the key determinant in ending the epoch of high-mass star formation (Omukai 2000; Bromm et al. 2001a). These authors find that very massive stars can no longer form once the gas is enriched to a level in excess of $Z_{\text {crit }} \simeq 10^{-3.5} \mathrm{Z}_{\odot}$. The 
criterion for the second transition in the cosmic star formation history is then

$$
Z_{\mathrm{IGM}}\left(z_{2 \rightarrow 3}\right) \sim Z_{\text {crit }}
$$

In Figure 4, we show that this condition results in the approximate range, $15 \lesssim z_{2 \rightarrow 3} \lesssim 20$, with the limits corresponding to the two assumptions for the mixing efficiency. In a somewhat different context, Schneider et al. (2002) have carried out a similar calculation, finding a transition redshift, $z \gtrsim 20$, that is broadly consistent with our estimated range.

In reality, not all locations in the universe will have a metallicity close to the mean value calculated in equation (6). In fact, the gas in a star forming halo is likely to be rapidly enriched to a level $\sim Z_{\text {crit }}$ locally (see $\S 3.3$ ), in contrast to the rather slow enrichment of the general IGM. A substantial fraction of the Pop III metal production, however, is expected to escape from the shallow DM potential wells at high redshifts (e.g., Madau et al. 2001; Mori et al. 2002). In addition, the enriched gas has to travel much shorter distances between neigboring halos at these early times, and it might therefore have been easier to establish a uniform metal distribution in the IGM. The mean IGM metallicity in equation (6) would then indeed be a good indicator of when the universe as a whole will undergo the transition from predominantly Pop III to predominantly Pop II star formation. Clearly, a more realistic understanding of how the metals are mixed, and to what degree of homogeneity, can only be gained from numerical simulations.

In addition to the metal enrichment due to supernovae, the IGM could also be enriched due to winds from the first stars. The importance of such winds, however, is predicted to be small in the absence of metals and dust grains in the stellar atmosphere (e.g., Kudritzki 2002).

\subsection{Implications}

The sequence of star-forming modes proposed in this section is expected to apply equally to an individual halo (going through different 'stages' of star formation), and in a statistical sense to the universe as a whole (going through different 'epochs'). It will be very interesting to determine the degree of synchronization in the star-formation modes between spatially disjoint regions of the universe. Is it possible that stars in a given location form in a different mode from that in a neighboring region? Or is the VMS feedback sudden enough to coordinate the star formation activity in a global fashion? We emphasize that answers to these important questions must await the results from full numerical simulations, and cannot be obtained from the simple analytical model presented in this paper.

By examining Figure 4, it appears that the IGM could reach a level of metal enrichment 


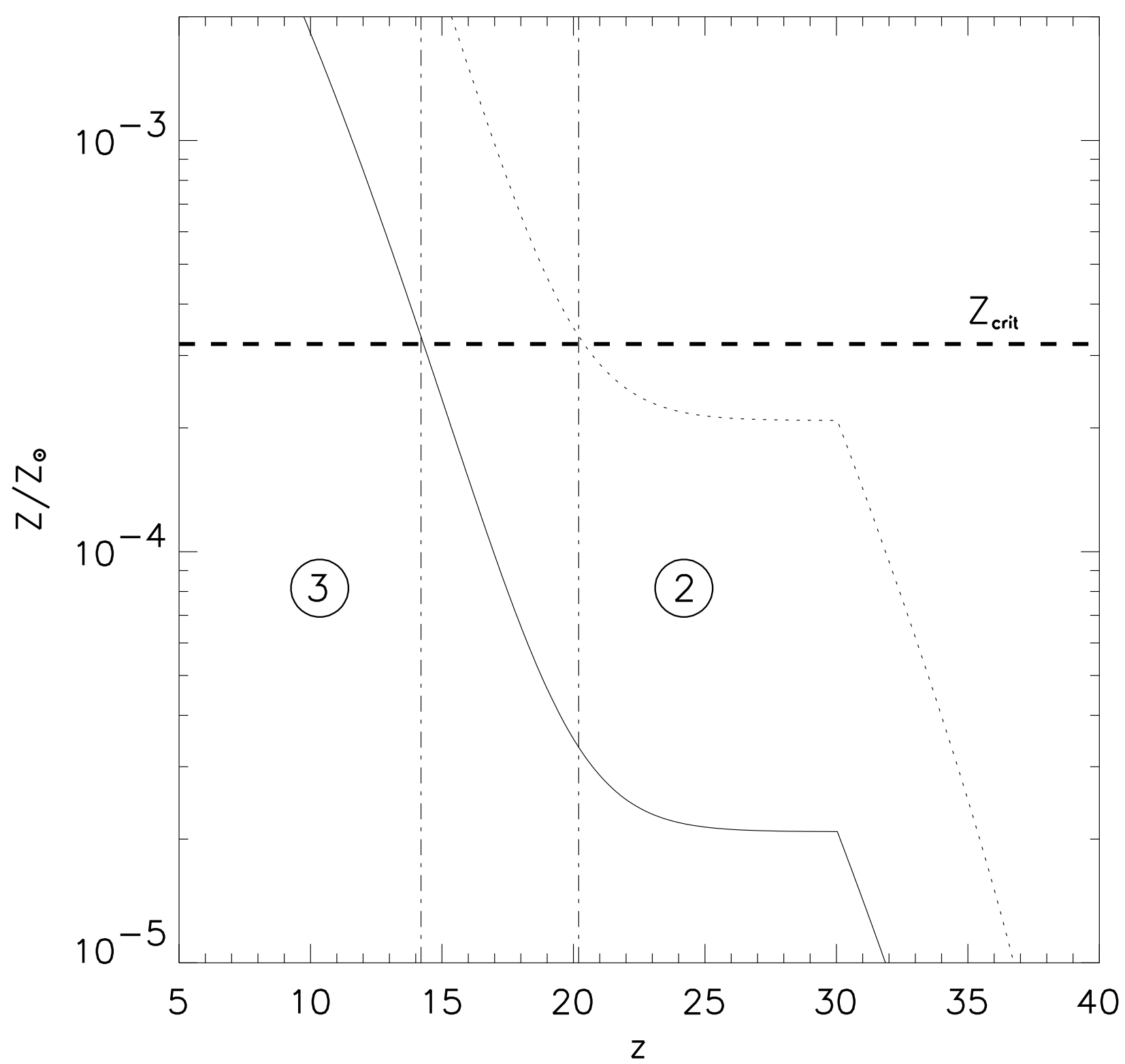

Fig. 4.- The transition from epoch 2 to 3 . IGM metallicity in units of $\mathrm{Z}_{\odot}$ as a function of redshift. The metallicity is calculated with the star formation history in Fig. 1, assuming a heavy element yield of $50 \%$, and two different mixing efficiencies. Solid line: Metal enrichment for $f_{\text {mix }}=0.05$. Dotted line: Metal enrichment for $f_{\text {mix }}=0.5$. Horizontal dashed line: The critical metallicity above which massive stars are no longer able to form. Vertical dot-dashed lines: The redshift at which the IGM metal enrichment reaches $Z_{\text {crit }}$, marking the transition from the second to the third epoch of star formation at high $z$. These two lines bracket the plausible range, $15 \lesssim z \lesssim 20$, where the transition is likely to occur. 
from VMS formation which is already close to that inferred for the low-column density Ly $\alpha$ forest at $z \sim 5$ (Songaila 2001). Within the context of our model, the IGM would have been enriched with a 'bedrock' of metals, corresponding to $Z_{\text {IGM }} \sim Z_{\text {crit }}$, already at $z_{2 \rightarrow 3} \gtrsim 15$. Again, a more realistic assessment of this hypothesis requires sophisticated numerical simulations.

\section{Stellar Populations at High z}

\subsection{Three Basic Populations}

As a consequence of our picture for high redshift star formation, we predict three distinct stellar populations at $z \gtrsim 5$ (see Table 1 ). VMSs form out of gas with $Z<Z_{\text {crit }}$ and constitute the 'classic' Population III. Low-mass stars, with typical masses of $\sim 1 \mathrm{M}_{\odot}$, that form out of already enriched gas with $Z>Z_{\text {crit }}$ make up Population II. These stars are actually observed, e.g., in the halo of our Galaxy. Finally, we propose a mechanism for producing a

novel Population II.5: intermediate-mass stars, forming out of gas with $Z<Z_{\text {crit }}$, that owe their existence to the mechanical, SN-driven feedback from VMSs.

\subsection{The Formation of Population II.5 Stars}

We assume that the immediate progenitor of a star is a centrally concentrated, selfgravitating gaseous clump with a mass close to the Bonnor-Ebert value (e.g., Palla 2002)

$$
M_{\mathrm{BE}} \simeq 700 \mathrm{M}_{\odot}\left(\frac{T}{200 \mathrm{~K}}\right)^{3 / 2}\left(\frac{n}{10^{4} \mathrm{~cm}^{-3}}\right)^{-1 / 2} .
$$

Here, the temperature and density are normalized to the characteristic values that follow from the microphysics of $\mathrm{H}_{2}$ cooling (see Bromm et al. 2002). Depending on the details of how the gas is accreted onto the nascent hydrostatic core in the center of the gravitationally unstable clump (e.g., Omukai \& Palla 2001; Ripamonti et al. 2002), the resulting stellar mass is expected to be somewhat smaller. We take this uncertainty into account by expressing the final stellar mass as $M_{*} \simeq \alpha M_{\mathrm{BE}}$, and choose the efficiency to be $\alpha \sim 0.3$. This value is close to that inferred for the formation of stars in the present-day universe (McKee \& Tan 2002). For Pop III stars we then have $M_{*} \gtrsim 200 \mathrm{M}_{\odot}$, in agreement with the results from numerical simulations. Notice that the effective star formation efficiency introduced in $\S 2.1$ is given by $\eta_{*} \simeq f_{\text {cool }} \alpha$, where $f_{\text {cool }}$ is the fraction of the gas that is incorporated into the cold, dense clumps. 
As pointed out in $\S 2.3$, the primordial gas cannot cool below $\sim 200 \mathrm{~K}$ as long as $Z<Z_{\text {crit }}$. A decrease in the stellar mass, therefore, has to rely on achieving higher densities, beyond the value of $\sim 10^{4} \mathrm{~cm}^{-3}$ that characterizes Pop III star formation. We propose that such a boost in density can naturally occur in the cooled, dense shell behind a SN-driven shock wave, in the following way.

An exploding VMS drives a blast-wave into the surrounding medium that shock-heats the gas to a post-shock temperature approximately given by $T_{\mathrm{ps}} \simeq\left(m_{\mathrm{H}} / k_{\mathrm{B}}\right) u_{\mathrm{sh}}^{2}$, where $u_{\mathrm{sh}}$ is the (time-dependent) shock speed. Initially, the shock will evolve adiabatically with only a modest increase in density, $n_{\mathrm{ps}} / n_{0} \leq(\gamma+1) /(\gamma-1)=4$ for $\gamma=5 / 3$. Here, $n_{0}$ is the density of the undisturbed, preshock gas. Eventually, however, the shocked gas will be able to cool and, evolving roughly isobarically (e.g., Shapiro \& Kang 1987; Yamada \& Nishi 1998), reach much higher compression factors.

To assess whether the post-shock gas can cool sufficiently, we have implemented a simple one-zone model, solving for the thermal and chemical evolution in a homogeneous parcel of gas. The chemical reaction network and the cooling processes pertaining to primordial gas are described in Bromm et al. (2002). Under the assumption of isobaricity, the density evolves according to $n \simeq n_{0} T_{\mathrm{ps}} / T$. Assuming that the post-shock gas is initially fully ionized, the only free model parameters are $n_{0}$ and $u_{\mathrm{sh}}$. In Figure 5 , we show the resulting temperature evolution for $n_{0}=10^{2} \mathrm{~cm}^{-3}$, characteristic of the gas in the vicinity of a VMS (see Bromm et al. 2002), and $u_{\mathrm{sh}}=200 \mathrm{~km} \mathrm{~s}^{-1}$, corresponding to $T_{\mathrm{ps}} \simeq 5 \times 10^{6} \mathrm{~K}$. In this case, the gas cools to $T_{f} \sim 200 \mathrm{~K}$ within $t \leq 10^{-2} t_{H}(z=20) \sim 10^{6} \mathrm{yr}$, which is shorter than the lifetime of a VMS. The overall compression in the case of Figure 5 is then $n_{f} \simeq n_{0} T_{\mathrm{ps}} / T_{f} \simeq 10^{4} n_{0}$. In general, the characteristic mass of a Pop II.5 star can be written as

$$
M_{*}(\mathrm{II} .5) \simeq 10 \mathrm{M}_{\odot}\left(\frac{n_{0}}{10^{2} \mathrm{~cm}^{-3}}\right)^{-1 / 2}\left(\frac{u_{\mathrm{sh}}}{200 \mathrm{kms}^{-1}}\right)^{-1} .
$$

This expression implicitly assumes that the post-shock gas is able to cool to $T_{f} \sim 200 \mathrm{~K}$ in a time that is short compared to the Hubble time. This requirement constrains the shock speed to be $u_{\mathrm{sh}} \lesssim 300 \mathrm{kms}^{-1}$. The characteristic mass of a Pop II.5 star is consequently reduced by at least an order of magnitude compared to the Pop III case.

Within our model, the formation of Pop II.5 stars is directly linked to the death of a Pop III VMS. An important quantity then is the efficiency, $\eta$, of the formation process which we define as the mass ratio of intermediate mass (Pop II.5) stars to very massive (Pop III) stars

$$
\eta \equiv \frac{M_{\mathrm{II} .5}}{M_{\mathrm{III}}} \sim \frac{\epsilon \Delta M_{\text {shell }}}{M_{*}(\mathrm{III})}
$$

where $\Delta M_{\text {shell }}$ is the mass of the cooled, dense shell, $\epsilon$ the efficiency of forming stars out of 
this material, and $M_{*}(\mathrm{III}) \sim 250 \mathrm{M}_{\odot}$ the typical mass of a Pop III star. We assume that roughly one-half of the mass that has been swept up by the end of the energy-conserving (Sedov-Taylor) phase ends up in the dense shell

$$
\Delta M_{\text {shell }} \sim 0.5 \frac{4 \pi}{3} R^{3} \rho_{0} \sim 0.5 \frac{E_{0}}{u_{\mathrm{sh}}^{2}},
$$

where $E_{0} \sim 10^{53} \mathrm{erg}$ is the kinetic energy of a PISN (Fryer et al. 2001). Taking the shock velocity at the onset of the radiative phase to be $u_{\mathrm{sh}} \sim 300 \mathrm{~km} \mathrm{~s}^{-1}$, we thus estimate $\Delta M_{\text {shell }} \sim 10^{4.5} \mathrm{M}_{\odot}$. In equation (10), we conservatively assume that only a small fraction of the dense material in the shell is available to form stars, $\epsilon \sim 0.01$, and we therefore predict the efficiency of forming Pop II.5 stars to be small: $\eta \sim 10^{2} \epsilon \sim 1$.

We would like to stress that the considerations presented here can only serve to physically motivate the possibility of such an intermediate stellar population. To fully explore the viability of our proposal, we plan to investigate the propagation of a VMS SN with numerical simulations (for numerical work on shock-induced star formation in the present-day universe, see Vanhala \& Cameron 1998 and references therein).

\subsection{Implications}

We propose that the three stellar populations discussed here can form only in certain of the epochs introduced in $\S 2$. According to our metallicity criterion, Pop III star formation occurs exclusively during the first two epochs. As we have argued above, the formation of Pop II.5 stars is induced by the death of a Pop III star. Only the second epoch, however, is conducive to such a triggered star formation mechanism. In epoch 1 , Pop III stars form in low-mass halos that are already severely disrupted by a single PISN. It is only later, in epoch 2 of the cosmic star formation history, that the then substantially more massive halos can survive a VMS explosion, and consequently enable the triggered formation of intermediatemass, Pop II.5 stars. To see this more clearly, consider the binding energy of a halo of mass $M$, collapsing at redshift $z$ (Barkana \& Loeb 2001):

$$
E_{b} \simeq 10^{54} \operatorname{erg}\left(\frac{M}{10^{8} \mathrm{M}_{\odot}}\right)^{5 / 3}\left(\frac{1+z}{25}\right)
$$

Using equation (1), we evaluate this expression for $M \simeq M_{\text {crit }}(z \simeq 25)$, resulting in

$$
E_{b} \simeq \begin{cases}10^{49} \mathrm{erg} & \text { for } \mathrm{H}_{2} \text { cooling } \\ 10^{53} \mathrm{erg} & \text { for } \mathrm{H} \text { cooling }\end{cases}
$$




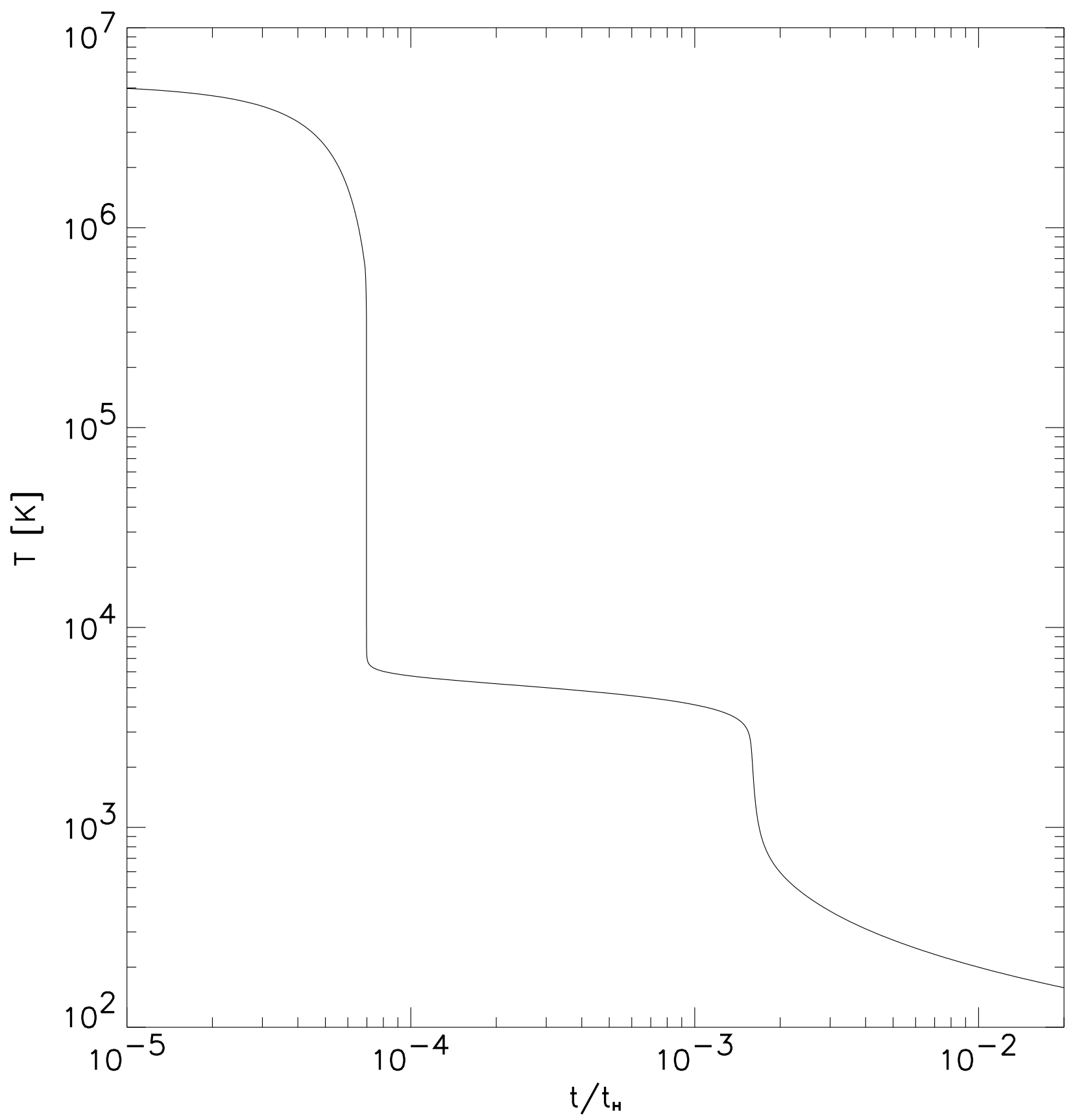

Fig. 5.- Thermal evolution in shocked gas. Gas temperature vs. time (in units of the Hubble time at $z \simeq 20$ ). Here, the shock has a velocity of $u_{\mathrm{sh}}=200 \mathrm{~km} \mathrm{~s}^{-1}$, and propagates into a medium with density $n_{0}=10^{2} \mathrm{~cm}^{-3}$. The initial, very sudden decrease in temperature is due to the onset of atomic ( $\mathrm{He}$ and $\mathrm{H}$ ) line cooling. The subsequent drop is brought about by molecular $\left(\mathrm{H}_{2}\right)$ cooling. It is evident that the shocked gas is able to cool to $\sim 200 \mathrm{~K}$ within a small fraction of the Hubble time at $z=20$. 
It is evident that the criterion for the formation of Pop II.5 stars, $E_{b} \gtrsim E_{0} \sim 10^{53} \mathrm{erg}$, is fulfilled in halos that cool via atomic hydrogen lines, but not in those that cool via $\mathrm{H}_{2}$.

Within the framework presented in this paper, Pop II stars can clearly form in epoch 3 , at which point we assume that most of the IGM is enriched to a level in excess of $Z_{\text {crit }}$. It is likely, however, that Pop II star formation already ensues during epoch 2. The metal enrichment due to VMSs is so efficient that the host system is expected to reach the critical metallicity well before the general IGM does. In fact, one can estimate that already one single VMS could enrich the $\sim 10^{7} \mathrm{M}_{\odot}$ of gas in the typical star-forming halo in epoch 2 to the critical level:

$$
\begin{aligned}
Z_{\text {halo }} & =\frac{M_{Z}}{M_{B}} \\
& =\frac{\sim 10^{2} \mathrm{M}_{\odot}}{\sim 10^{7} \mathrm{M}_{\odot}} \sim 10^{-3.3} \mathrm{Z}_{\odot} \gtrsim Z_{\text {crit }},
\end{aligned}
$$

where we have assumed for simplicity that none of the metals have escaped the halo, corresponding to the limiting case of $f_{\text {mix }}=0$ (see $\S 2.3$ ).

During epoch 2, therefore, we expect all three stellar populations to occur almost simultaneously in a given star forming system. In Table 1, we summarize the association of the various stellar populations with the distinct epochs of star formation.

We now tentatively suggest how these stellar populations might relate to the different classes of SNe as proposed by Qian \& Wasserburg (2002). These authors (henceforth QW) account for the observed abundance patterns in low-metallicity Galactic halo stars within the framework of three distinct classes of SNe. At the beginning of the nucleosynthetic chain, a generation of very massive stars explodes to enrich the primordial material mostly with Fe, but producing no elements beyond the iron peak. It seems natural to identify the QW VMS SNe with the Pop III PISNe discussed here. According to QW, at some later stage in the chemical enrichment history, more normal, type II, SNe begin to contribute nucleosynthetic products. More specifically, QW distinguish between a class of high-frequency and lowfrequency type II SNe, named $\mathrm{SN} \mathrm{II}(H)$ and $\mathrm{SN} \operatorname{II}(L)$, respectively. The $H$ events produce mainly the heavy $r$-process elements but no $\mathrm{Fe}$, whereas the $L$ events contribute mainly the light $r$-process elements as well as Fe. These two classes of SN II are postulated on purely phenomenological grounds, and it is presently not known what kind of progenitor stars could physically give rise to them. There are, however, some indications that the $H$ events correspond to the explosion of comparatively low-mass stars that are only barely able to reach the SN stage (see QW and references therein). Given that Pop II.5 stars are more massive than Pop II stars by one order of magnitude, we speculate that Population II.5 might predominantly give rise to $\mathrm{SNe} \operatorname{II}(L)$, whereas Population II could lead to both the $H$ 
and $L$ events. Again, we summarize these proposed identifications in Table 1.

Regardless of the detailed correspondence discussed above, our general model for the character of star formation at high redshift seems to provide a promising astrophysical context for the QW proposal. More work, both observational and theoretical, is required to explore this possible connection further.

\section{Observational Consequences}

\subsection{Supernova Rates as Star Formation Tracer}

\subsubsection{Modeling}

The cosmic supernova rate as a function of redshift is potentially an excellent tracer of the different modes of star formation envisaged in this paper. A number of authors have previously advocated this method of tracing star formation, predicting supernova rates based on the metallicity of the IGM at high redshifts (Miralda-Escudé \& Rees 1997), or on the empirically determined star formation rate out to $z \sim 5$ (e.g., Madau, Della Valle \& Panagia 1998; Sadat et al. 1998; Dahlén \& Frannson 1999). In our study, we use theoretical estimates of the cosmic star formation rate to predict the number of supernovae from VMSs and from regular stars (Pop II) out to much higher redshifts. Observational determinations of these rates will constrain the three epochs of star formation which we are proposing. Including observational selection functions is beyond the scope of this paper. We will, however, present rough estimates of the flux limit and wavelength coverage needed in order to observe SN explosions at high redshift. The observational capability of NGST has recently been studied for high- $z$ type II SNe (Dahlén \& Frannson 1999), and in a preliminary fashion for PISNe from VMSs (Heger et al. 2002).

We use the results of recent cosmological simulations by Springel \& Hernquist (2002) to model the Pop II star formation history of the universe. These simulations agree roughly with observations of the star formation rate out to $z \sim 5$, but extend to much higher redshifts than are currently probed by direct observations, and also reproduce the mean density of stars in the universe. These authors provide a simple analytical fit to the SFR as follows:

$$
\psi_{*}(z ; \mathrm{II})=K \frac{b \exp \left[a\left(z-z_{m}\right)\right]}{b-a+a \exp \left[b\left(z-z_{m}\right)\right]},
$$

where the normalization is $K=0.15 \mathrm{M}_{\odot} \mathrm{yr}^{-1} \mathrm{Mpc}^{-3}$ and the fitting parameters are $a=3 / 5$, $b=14 / 15$, and $z_{m}=5.4$. The time delay between the formation and explosion of a massive star is $<20 \mathrm{Myr}$, and therefore the Type II supernova rate should trace the star formation 
rate almost exactly. For a Salpeter IMF, there is one Type II supernova (SN II) per $\sim 150 \mathrm{M}_{\odot}$ of star formation, and we consequently divide the SFR by this factor (which we call $M_{\mathrm{SN}}$ (II) ) to obtain the Pop II SN rate. Hernquist \& Springel (2002) find that the SFR at $z<z_{m}$ in their simulations actually has a power-law dependence on the expansion rate, rather than evolving exponentially with redshift; however the fit given in the above equation is sufficient for our present purposes.

For the PISN rate we use the VMS star formation rate in Figure 2. The typical lifetime of a VMS is $\sim 3 \mathrm{Myr}$ so we can again assume the SN rate traces the SFR closely. We take typical VMSs to have masses of $250 \mathrm{M}_{\odot}$ and assume they all die as supernovae to calculate the VMS supernova rate (i.e. $M_{\mathrm{SN}}(\mathrm{III})=250 \mathrm{M}_{\odot}$ ). VMSs are predicted to end their lives as pair-instability supernovae if $M<260 \mathrm{M}_{\odot}$, and these should be very energetic and luminous events. Bear in mind, however, that the mass function, and hence end state of Pop III stars is still somewhat uncertain, making this supernova rate a crude estimate.

We also calculate the rate of Pop II.5 supernovae, using the result from the last section that the Pop II.5 SFR is directly proportional to the Pop III SFR, with constant of proportionality $\eta \sim 1$. Due to the uncertain nature of the Pop II.5 IMF we must make simple assumptions to get the mass in stars formed per supernova. The characteristic stellar mass was shown to be about $10 \mathrm{M}_{\odot}$, so we assume that half of the stellar mass goes into stars that can explode. We further assume that the average mass of a Pop II.5 supernova progenitor is $\sim 20 \mathrm{M}_{\odot}$. From this we find that $M_{\mathrm{SN}}($ II. 5$) \sim 40 \mathrm{M}_{\odot}$ for Pop II.5 supernovae. We expect these supernovae to be of Type II, although with very low metallicity.

We convert these rates per unit volume into rates observed per unit time and per unit redshift interval:

$$
\begin{aligned}
\frac{\mathrm{d} N}{\mathrm{~d} t_{o b s} d z} & =\frac{\mathrm{d} N}{(1+z) \mathrm{d} t_{e m} \mathrm{~d} V} \frac{\mathrm{d} V}{\mathrm{~d} z} \\
& =\frac{1}{M_{\mathrm{SN}}} \frac{\psi_{*}(z)}{(1+z)} \frac{c}{H_{0}} \frac{4 \pi f_{\text {sky }} r^{2}(z)}{\sqrt{\Omega_{m}(1+z)^{3}+\Omega_{\Lambda}}}
\end{aligned}
$$

Here $\mathrm{d} V$ is a comoving volume element, $M_{\mathrm{SN}}$ is the mass in stars formed per supernova for the relevant stellar population, $f_{s k y}$ the fraction of the sky observed, and $c / H_{0}$ the Hubble length. Note that the Pop II.5 and Pop III supernova rates differ only in their respective values for $M_{*}$ calculated above. The respective time intervals in the observer and emitter frames, $\mathrm{d} t_{o b s}$ and $\mathrm{d} t_{e m}$, are related by the factor of $(1+z)$ in the denominator. The comoving distance to redshift $z$ is

$$
r(z)=\frac{c}{H_{0}} \int_{0}^{z} \frac{\mathrm{d} z^{\prime}}{\sqrt{\Omega_{m}\left(1+z^{\prime}\right)^{3}+\Omega_{\Lambda}}} .
$$




\subsubsection{Results}

The results for our cosmological parameters are shown in Figure 6, where we plot the number of supernovae that could be observed per year and per square degree. Note that this figure does not incorporate magnitude limits or observational selection functions, but does simply show the predicted number of supernovae that can in principle be observed.

The redshift distribution of SNe follows the star formation rate, peaking at $z \sim 5$ for Pop II, and showing the same break at $z=30$ for PISNe as in Figure 2. We expect the transition from predominantly PISNe to Pop II SNe to occur around a redshift of $z \sim 15-20$ (see Figure 4), and we therefore show the rates for both types of supernova in this redshift range. As this transition occurs, we expect the Pop II SN rate to gradually drop below the solid line towards higher redshifts as Pop II star formation switches off. Similarly we expect the Pop III (Pop II.5) rate to drop below the dashed (dotted) line towards lower redshifts as Pop III star formation ceases. We do not expect any VMSs at $z \lesssim 15$, but we extend our calculation down to $z=7$ because the reionization of the universe at roughly this redshift provides a robust lower limit for the end of VMS formation (e.g., Oh et al. 2001). That the PISN and Pop II SN rates are so similar at the transition redshifts is because the star formation rate for Pop III at $z \sim 15$ which we predict analytically is similar to the rate (for Pop II) found in the simulations of Springel \& Hernquist (see Figure 1). Again, we emphasize that the break in the VMS supernova rate at $z=30$ is artificially sharp, reflecting the abrupt transition in the underlying star formation rate.

In terms of total numbers of supernovae, we predict $\sim 2 \times 10^{6}$ PISNe per year over the whole sky (above $z=15$ ), or about 50 per square degree per year. This still comprises only $\sim 0.4 \%$ of all supernovae, as we find $\sim 4 \times 10^{8}$ SNeII per year (all sky), a value comparable to previous estimates (Miralda-Escudé \& Rees 1997; Madau et al. 1998). This number can be understood by a simple order of magnitude estimate as follows. About $10 \%$ of the baryons in the universe are in stars by the present time, giving a local density in stars of $\rho_{*} \simeq 0.1 \Omega_{b} \rho_{\text {crit }} \sim 6 \times 10^{8} \mathrm{M}_{\odot} / \mathrm{Mpc}^{3}$ for our cosmological parameters (Peebles 1993). The size of the observable universe is $\sim 10 \mathrm{Gpc}$ out to $z=5$ (most of the star formation happens later than $z=5$ because there is very little physical time before this), so the total mass in stars in this volume, ignoring redshift evolution effects, is $\sim 10^{21.5} \mathrm{M}_{\odot}$. About $10 \%$ of the mass in stars goes into stars which can explode, so the mass in supernovae is $\sim 10^{20.5} \mathrm{M}_{\odot}$. The mass weighted average for a supernova progenitor's mass is $\sim 30 \mathrm{M}_{\odot}$, and the age of the universe is 14 Gyr. The number of supernovae per year is then

$$
\frac{\mathrm{d} N}{\mathrm{~d} t_{\mathrm{obs}}} \sim \frac{10^{20.5}}{30 \times 14 \times 10^{9} \mathrm{yr}} \sim 7 \times 10^{8}{\mathrm{SNe} \mathrm{yr}^{-1}}
$$

similar to our numerical integration of the calculated SN rate. All calculations of the SN 


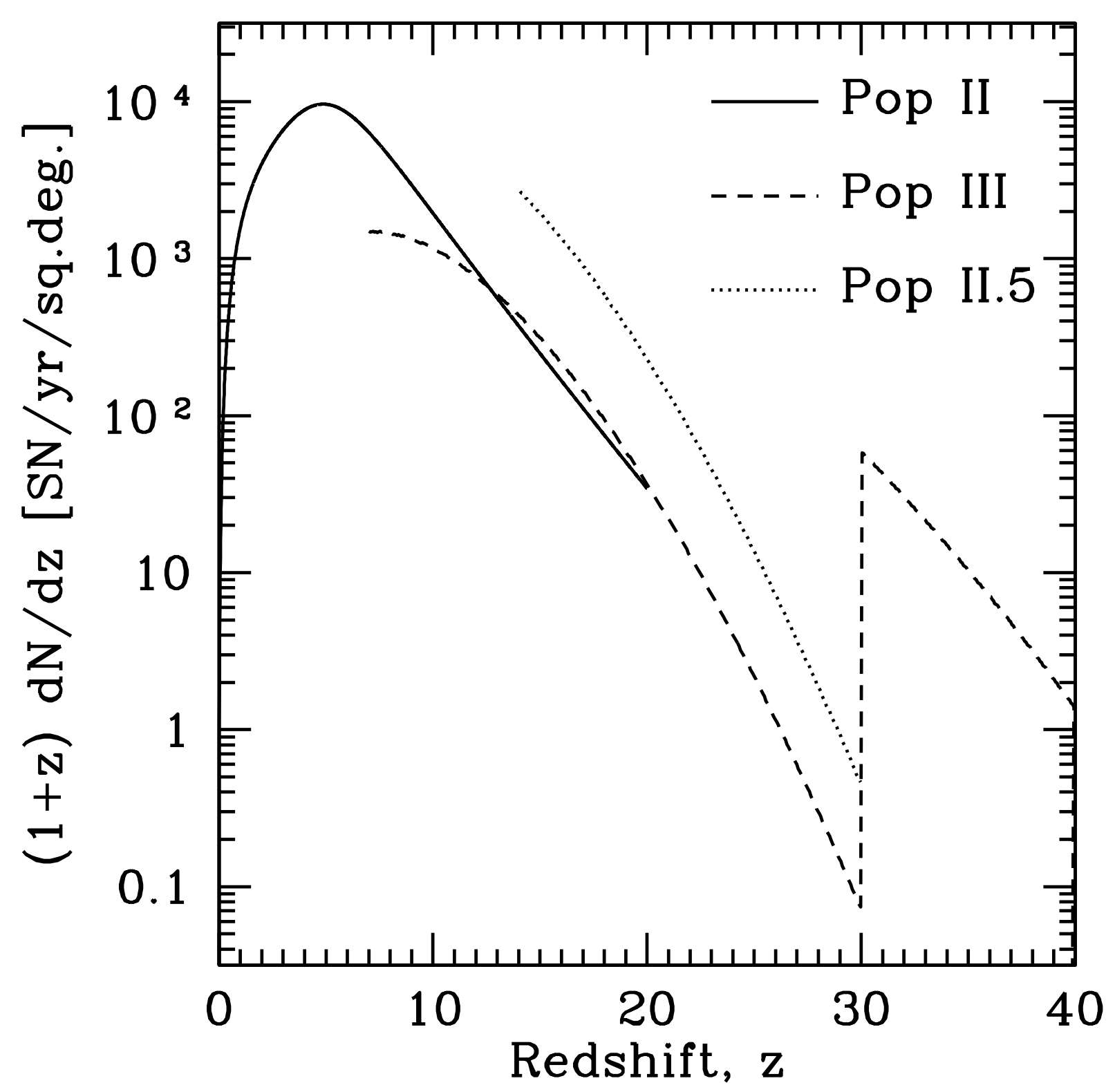

Fig. 6.- Supernova rate as a function of redshift for Type II and VMS supernovae. Pop II and Pop II.5 refer to Type II supernovae from Pop II and Pop II.5 stars, respectively. Pop III refers to VMS supernovae. The assumed star formation rates are detailed in the text. We show the number of supernovae per observed year in one square degree of the sky, plotted as a function of redshift. 
rate based on the cosmic SFR are expected to yield an answer around this value.

\subsubsection{Observational Prospects}

We now address the question of whether any of these supernovae at very high redshift could be observed in the foreseeable future. Type Ia supernovae have a peak B-magnitude of $M_{B} \simeq-19$, with Type II supernovae being about 2.5 magnitudes fainter (e.g., Filippenko 1997). VMSs are predicted to end their lives as pair instability supernovae if their mass is in the range $140 \mathrm{M}_{\odot}<M<260 \mathrm{M}_{\odot}$ (Heger \& Woosley 2002), but they have not yet been observed and their luminosity is not well known. Heger \& Woosley (2002) have shown that they can produce large masses of ${ }^{56} \mathrm{Ni}$ during supernova nucleosynthesis. If the light curve is powered by ${ }^{56} \mathrm{Ni}$ decay, then these supernovae should be brighter than Type Ia's by a large factor (the ratio of their ${ }^{56} \mathrm{Ni}$ production). Preliminary results from Heger et al. (2002) show that reality is somewhat more complicated, and these authors find that a PISN is only a factor of a few brighter than a type Ia. Because of this uncertainty in luminosity, we will calculate the apparent brightness of a type Ia SN at large redshifts, and scale this up for the brighter PISN. The apparent brightness in a flat universe is given by

$$
f_{\nu}(z)=\frac{L_{(1+z) \nu}}{4 \pi(1+z) r^{2}(z)},
$$

where $L_{\nu}$ is the monochromatic luminosity of the source at redshift $z$. For $z=20, M_{B}=-19$, and our cosmological parameters we obtain

$$
f_{\nu}\left(\lambda_{\mathrm{obs}} \simeq 9 \mu \mathrm{m}, z=20\right) \simeq 5 \mathrm{nJy}
$$

The observed wavelength of $9 \mu \mathrm{m}$ is the redshifted $B$-band. If the flux does not drop off too much in the rest frame near-UV, then there should be a similar flux level in the nearIR $(\lambda<5 \mu \mathrm{m})$ where NGST should have sub-nJy sensitivity (Barkana \& Loeb 2001). This indicates that even a Type Ia SN should be observable with NGST out to $z \simeq 20$, and PISNe should be easily observable if they are at least a few times brighter. Type II supernovae, on the other hand, will be very hard to detect at these high redshifts because they are typically a factor of 10 fainter. For this reason, even though we predict Pop II.5 supernovae to be more numerous than PISNe, we expect that they will not be nearly as useful as observational probes of the high redshift universe. Of course observing them would be useful for assessing the efficiency of low mass star formation, and for testing our shock compression model.

Marri \& Ferrara (1998) have discussed the feasibility of using gravitational lensing mag-

nification due to intervening mass concentrations to observe high redshift supernovae more 
easily, and potentially bring even type II supernovae within reach of NGST at $z \gtrsim 10$. Similar considerations also apply to our calculations, and this may help in obtaining detailed observations of PISNe. It is important to be able to identify them reliably, distinguish them from other types of supernova, and measure their redshift if we are to use them for cosmological purposes. To be able to do this, we need a sample of SNe that are bright enough for spectroscopic observations. Since none have been observed locally, we need the additional lensing magnification to investigate SNe in detail at high redshift.

\subsection{Metal Poor Halo Stars}

If our mechanism for producing extremely low metallicity, low mass stars (Pop II.5 in our terminology) via shock compression occurs in reality, then some of these stars should still be present in the halo of our galaxy. They would of course be very old ( $\sim 14$ Gyr $)$, so only the lowest mass stars with $M \lesssim 0.8 \mathrm{M}_{\odot}$ would remain (Girardi et al. 2000; Siess, Livio \& Lattanzio 2002). We can use our model to predict how many such stars we would expect to find in a typical Milky Way-sized halo and, given a model for the stellar halo density profile, their number density in the solar neighborhood (we use a similar approach to that of Hernandez \& Ferrara 2001). Significant uncertainties in this calculation are the efficiency of producing Pop II.5 stars (as discussed in §3.2), the fraction of them that end up in the halo as opposed to the bulge, and their mass function.

The star formation rate of Pop II.5 stars is given by $\psi_{*}\left(z ;\right.$ II.5) $=\eta \psi_{*}(z ;$ III $)$ in the redshift range when they form. There is a slight difference to the way $\psi_{*}(z ;$ III $)$ is calculated for this problem, in that we are interested in the stars that end up in the Milky Way (MW) halo. Therefore, instead of the usual Press-Schechter mass function in the Santos et al. (2002) model, we use the Extended Press-Schechter mass function of halos at redshift $z$ which will end up in a halo the mass of the MW at $z=0$ (Lacey \& Cole 1993).

For a MW mass (total) of $M_{\mathrm{MW}}=10^{12} \mathrm{M}_{\odot}$ we find that $10^{7.5} \mathrm{M}_{\odot}$ of Pop III stars are formed in MW progenitor halos, over the redshift range from $z=30$ to $z=15$ in which we expect Pop II.5 stars to form. Interestingly, because the metal yield of PISNe is $\sim 0.5$, this results in a MW metal enrichment of

$$
Z \sim 0.5 \times\left(\frac{10^{7.5} \mathrm{M}_{\odot}}{10^{12} \mathrm{M}_{\odot}}\right)\left(\frac{\Omega_{m}}{\Omega_{b}}\right)\left(\frac{\mathrm{Z}_{\odot}}{0.02}\right) \sim 10^{-2} \mathrm{Z}_{\odot},
$$

similar to the metallicity of the lowest metallicity globular clusters and thick disk stars $([\mathrm{Fe} / \mathrm{H}]=-2.2$, Freeman \& Bland-Hawthorn 2002).

For a given value of $\eta$, this mass in Pop III stars gives us the corresponding mass in 
Pop II.5 stars:

$$
M_{\mathrm{II} .5} \simeq 10^{7.5} \mathrm{M}_{\odot}\left(\frac{\eta}{1.0}\right)\left(\frac{\eta_{*}}{0.1}\right) .
$$

We now wish to convert this into the number density of Pop II.5 stars that could be observable in the MW halo today. First, we have argued that the characteristic mass of Pop II.5 stars is $\sim 10 \mathrm{M}_{\odot}$, so most of these stars will have died long before the present. We parameterize this in a very simple way by assuming that some fraction, $f_{\mathrm{imf}} \sim 0.1$ of the mass in stars formed goes into stars with $M<0.8 \mathrm{M}_{\odot}$. A second poorly constrained process is where in the MW these stars reside. It is expected that most of the earliest forming stars will be incorporated into the bulge of the MW, with a small fraction in the halo (White \& Springel 2000). We introduce $f_{\text {halo }} \sim 0.1$ as the fraction of Pop II.5 stars which end up in the halo. Finally we make the reasonable assumption that most of the Pop II.5 stars remaining today are near the end of their lives (i.e. their mass function is increasing to higher masses at $M<1 \mathrm{M}_{\odot}$ ), so we get the number of halo stars by dividing their total mass by $0.8 \mathrm{M}_{\odot}$. This gives us the total number of Pop II.5 stars in the MW halo:

$$
N_{\text {II. } 5} \sim 10^{5.5} \mathrm{M}_{\odot}\left(\frac{\eta}{1.0}\right)\left(\frac{f_{\text {halo }}}{0.1}\right)\left(\frac{f_{\mathrm{imf}}}{0.1}\right) .
$$

To relate this to the observed number density of low metallicity halo stars we distribute the Pop II.5 stars in a halo with density profile $\rho(r)=\rho\left(r_{\odot}\right) r_{\odot}^{3} /\left(r_{c}^{3}+r^{3}\right)$, where the solar orbital radius, $r_{\odot}=8.0 \mathrm{kpc}$ (Reid 1993), is assumed much larger than the core radius, $r_{c}$, of the halo profile (needed for finite halo mass). This distribution only diverges logarithmically at large radii, so the mass of the halo is quite insensitive to the uncertain cutoff radius and core radius (we take $r_{\text {cut }} / r_{\text {core }}=300$ for definiteness). Using this profile, together with the total number of stars from the previous paragraph, the number density of Pop II.5 stars in the solar neighborhood is

$$
n_{\text {II. } 5} \sim 10 \text { stars } / \mathrm{kpc}^{3}\left(\frac{\eta}{1.0}\right)\left(\frac{f_{\text {halo }}}{0.1}\right)\left(\frac{f_{\text {imf }}}{0.1}\right) .
$$

We can make a crude comparison of this prediction with the results of observational searches for metal poor halo stars (e.g., Beers et al. 2000). Using their Figure 5, we see that most of their stars are within $2 \mathrm{kpc}$ of the sun, which we take to be the radius of their survey (covering 1940 square degrees of the sky). Also from that figure, they have $\sim 5$ stars with $[\mathrm{Fe} / \mathrm{H}] \leq-3.5$, and none with $[\mathrm{Fe} / \mathrm{H}] \leq-4.0$. If we identify these stars with Pop II.5, this gives them an observed space density of $n \sim 3 \mathrm{kpc}^{-3}$. This is similar to our theoretical estimate above, given the uncertainty associated with some of our parameters.

The observed stars all have a metallicity very close to our critical metallicity, so it is not clear if they are very low metallicity Pop II stars or if they were formed by our Pop II.5 
mechanism. We have shown that, with reasonable modeling assumptions, it is possible to produce these stars from primordial gas, and that their metallicity could be due to pollution from Pop III supernovae which triggered their formation (it could also be partly due to pollution from swept up gas as they travel through the galactic disk). Current observational searches are beginning to give us a clearer picture of the number of very low metallicity stars in the galactic halo, and we anticipate that this will continue with future observations. On the theoretical side, numerical simulations can in principle reduce the uncertainties in our Pop II.5 model by determining the values of $\eta, f_{\text {halo }}$, and possibly also of the IMF from the mass spectrum of collapsing clumps, although this is a very challenging problem to simulate accurately.

\section{Summary and Conclusions}

We have investigated the history of star formation in the high redshift universe, focusing on the role played by very massive Pop III stars. We have argued that this history is shaped by the various feedback effects exerted by those stars, resulting in three distinct epochs of star formation.

The first impact Pop III stars have on their surroundings is radiative, as their soft UV flux dissociates the molecular hydrogen in other minihalos (Haiman et al. 1997, 2000). Because of their copious UV emission, and the small molecular fraction $\left(\sim 10^{-4}-10^{-6}\right)$ in minihalos, this photodissociation is likely to happen quickly, and fairly completely. A numerical investigation of this process was performed by Machacek et al. (2001), who simulated this suppression of star formation in low mass halos by incorporating a uniform soft UV background into a cosmological simulation. While this assumption does not allow them to follow the detailed build-up of the UV radiation field as Pop III star formation switches on, it does give a good indication of the overall effect. It was found that the UV radiation can effectively suppress star formation due to molecular cooling in low mass halos, delaying their collapse from redshift $z \sim 30$ to $z \sim 20$.

It is encouraging that both analytic and numerical calculations agree quite well. In Figure 2, we find that once a significant UV radiation field has been set up by $z \sim 30$, further star formation is strongly inhibited until more massive halos start to form, and the SFR does not recover to its $z \sim 30$ value until $z \lesssim 20$. This is all in good agreement with the simulation results, except that Machacek et al. (2001) did find that more massive halos of $M \gtrsim 10^{7} \mathrm{M}_{\odot}$ were able to retain some molecular hydrogen. Hence, these halos were still able to cool via molecular lines, indicating that the SFR should not go all the way down to the atomic cooling curve, but it is likely to drop most of the way. Future simulations 
will be able to follow this dissociation process in detail, taking into account the effect of self-shielding, with a time-varying radiation field generated by point sources at the sites of Pop III star formation. In summary, the radiative feedback from Pop III stars results in the transition, at $z \sim 30$, between the first two epochs of star formation at high redshift.

The second impact Pop III stars have on surrounding material is chemical, and we identify this feedback as the main effect governing the transition from forming predominantly very massive stars to forming normal, lower mass ones (e.g., Omukai 2000; Nishi \& Tashiro 2000; Bromm et al. 2001a; Schneider et al. 2002). With the assumption that VMSs can only form in gas with a metallicity lower than a critical value, $Z_{\text {crit }} \sim 10^{-3.5} \mathrm{Z}_{\odot}$, we estimate that most of the star-forming material in the IGM has reached this level in the redshift range $z \sim 15-20$, which delineates the second transition in the star formation history, between epochs 2 and 3 .

An important issue is whether such a well-defined transition redshift between massive and more normal star formation actually occurs. This depends on how synchronized different parts of the universe are in crossing the critical metallicity threshold, which in turn depends on how well the supernova ejecta are mixed through the IGM at high redshifts. It has been suggested (Madau et al. 2001) that enrichment becomes more synchronized and uniform at higher redshifts. Dark matter halos are of much lower mass and have shallower potential wells, making it easier for metals to escape the halo. Supernovae from VMSs are predicted to be very energetic, with explosion energies significantly larger than the binding energy of halos at $z \gtrsim 20$ (Barkana \& Loeb 2001). Furthermore, the universe is much denser at high redshift and halos are consequently much closer together, again making it easier to pollute the IGM uniformly with metals. It is also possible, however, that enrichment at high redshifts is similar to that at low redshifts, where it has been shown to be very inhomogeneous and incomplete (e.g., Gnedin 1998; Cen \& Ostriker 1999; Aguirre et al. 2001a,b). If this were the case, the transition redshift at which the mean mass-weighted metallicity of the universe crosses the critical threshold would not be a very meaningful quantity. Which of these two cases is true is an important and interesting question, and can only be addressed theoretically by more detailed cosmological simulations which can resolve star-forming halos at high redshifts.

The demise of Pop III stars in SN explosions opens up the possibility for a new stellar population which we have termed Population II.5. These stars are characterized by masses, $M_{*} \sim 10 \mathrm{M}_{\odot}$, intermediate to that of Pop III and Pop II stars, and by extremely low metallicities, below the critical value that would normally lead to the formation of VMSs. This possibility relies on the gas in the vicinity of the SN being compressed in a radiative shock, leading to a significant increase in density, and thus possibly to a reduction of the fragment mass, $M_{\mathrm{BE}} \propto \rho^{-1 / 2}$, to values much lower than the one realized in primordial 
gas. During an intermediate epoch, therefore, low- and high-mass stars might form almost simultaneously. A similar idea had already been proposed in a prescient paper by Cayrel (1986), primarily to explain the so-called G-dwarf problem, i.e., the observed lack of metalpoor stars in our Galaxy (see also Kashlinsky \& Rees 1983; Nakamura \& Umemura 2001).

It is to be expected that the mass range of Pop II.5 stars extends down to $\lesssim 1 \mathrm{M}_{\odot}$, and these stars at the low-mass end of the Pop II.5 IMF should still be around today. Intriguingly and possibly providing a case in point, the recent discovery of the extremely metal poor star CS 29498-043 with $[\mathrm{Fe} / \mathrm{H}]=-3.7$ and a significant overabundance of $\mathrm{Mg}$ and $\mathrm{Si}$, has been tentatively interpreted as hinting at a new class of stars (Aoki et al. 2002). Ongoing surveys of extremely metal-poor halo stars, much improved in size and quality, should soon be able to test our prediction further.

Many of the uncertainties in our argument have to be addressed with detailed numerical simulations, and we plan to do so in future work. The framework presented in this paper does provide a coherent context for these numerical studies, and it highlights the important physical questions that define the challenge of elucidating the end of the cosmic "dark ages".

We are grateful to Andrea Ferrara, Alex Heger, Marc Kamionkowski, Robert Kirshner, Avi Loeb, Thomas Matheson, Mike Santos, Volker Springel, and Jerry Wasserburg for helpful discussions. We thank the anonymous referee for comments that improved the presentation of this paper. This work was supported in part by NSF grants ACI 96-19019, AST 98-02568, AST 99-00877, and AST 00-71019.

\section{REFERENCES}

Abel, T., Bryan, G. L., \& Norman, M. L. 2000, ApJ, 540, 39

Abel, T., Bryan, G. L., \& Norman, M. L. 2002, Science, 295, 93

Aguirre, A., Hernquist, L, Schaye, J., Katz, N., Weinberg, D.H., \& Gardner, J. 2001a, ApJ, 561,521

Aguirre, A., Hernquist, L, Schaye, J., Weinberg, D.H., Katz, N. \& Gardner, J. 2001b, ApJ, 560,599

Aoki, W., Norris, J.E., Ryan, S.G., Beers, T.C., \& Ando, H. 2002, ApJ, 576, L141

Ashman, K. M., \& Zepf, S. E. 1998, Globular Cluster Systems (Cambridge: Cambridge Univ. Press) 
Barkana, R., \& Loeb, A. 2001, Phys. Rep., 349, 125

Barkana, R. 2002, NewA, 7, 85

Beers, T.C., Chiba, M., Yoshii, Y., Platais, I., Hanson, R.B., Fuchs, B., \& Rossi, S. 2000, AJ, 119, 2866

Blain, A. W., \& Natarajan, P. 2000, MNRAS, 312L, 35

Bromm, V., Coppi, P. S., \& Larson R. B. 1999, ApJ, 527, L5

Bromm, V., Ferrara, A., Coppi, P. S., \& Larson, R. B. 2001a, MNRAS, 328, 969

Bromm, V., Kudritzki, R.P., \& Loeb A. 2001b, ApJ, 552, 464

Bromm, V., \& Clarke, C. 2002, ApJ, 566, L1

Bromm, V., Coppi, P. S., \& Larson, R. B. 2002, ApJ, 564, 23

Bromm, V., \& Loeb, A. 2002, ApJ, 575, 111

Cayrel, R. 1986, A\&A, 168, 81

Cen, R., \& Ostriker, J.P. 1999, ApJ, 519, L109

Ciardi, B., Ferrara, A., \& Abel, T. 2000a, ApJ, 533, 594

Ciardi, B., Ferrara, A., Governato, F., \& Jenkins, A. 2000b, MNRAS, 314, 611

Dahlén, T., \& Fransson, C. 1999, A\&A, 350, 349

Draine, B.T., \& Bertoldi, F. 1996, ApJ, 468, 269

Filippenko, A.V. 1997, ARA\&A, 35, 309

Freeman, K., \& Bland-Hawthorn, J. 2002, ARA\&A, 40, 487

Fryer, C.L., Woosley, S.E., \& Heger, A. 2001, ApJ, 550, 372

Fuller, T. M., \& Couchman, H. M. P. 2000, ApJ, 544, 6

Girardi, L., Bressan, A., Bertelli, G., \& Chiosi, C. 2000, A\&AS, 141, 371

Glover, S.C.O., \& Brand, P.W.J.L. 2001, MNRAS, 321, 385

Gnedin, N.Y. 1998, MNRAS, 294, 407 
Haiman, Z., Rees, M. J., \& Loeb, A. 1997, ApJ, 476, 458

Haiman, Z., Abel, T., \& Rees, M. J. 2000, ApJ, 534, 11

Heger, A., Woosley, S. E., Baraffe, I., \& Abel, T. 2002, in Lighthouses of the Universe, ESO Astrophys. Symp., ed. M. Gilfanov, R. Sunyaev, \& E. Churazov (Berlin: Springer), 369

Heger, A., \& Woosley, S. E. 2002, ApJ, 567, 532

Hernandez, X., \& Ferrara, A. 2001, MNRAS, 324, 484

Hernquist, L. \& Springel, V. 2002, MNRAS, submitted (astro-ph/0209183)

Kashlinsky, A., \& Rees, M. J. 1983, MNRAS, 205, 955

Kudritzki, R.P. 2002, ApJ, 577, 389

Lacey, C., \& Cole, S. 1993, MNRAS, 262, 627

Machacek, M.E., Bryan, G.L., \& Abel, T. 2001, ApJ, 548, 509

Madau, P., Della Valle, M., \& Panagia, N. 1998, MNRAS, 297, L17

Madau, P., Ferrara, A., \& Rees, M. J. 2001, ApJ, 555, 92

Marri, S., \& Ferrara, A. 1998, ApJ, 509, 43

McKee, C.F., \& Tan, J.C 2002, Nature, 416, 59

Miralda-Escudé, J., \& Rees, M.J. 1997, ApJ, 478, L57

Mori, M., Ferrara, A., \& Madau, P. 2002, ApJ, 571, 40

Nakamura, F., \& Umemura, M. 2001, ApJ, 548, 19

Nishi, R., \& Tashiro, M. 2000, ApJ, 537, 50

Oh, S.P., Nollett, K.M., Madau, P.,\& Wasserburg, G.J. 2001, ApJ, 562, L1

Oh, S. P., \& Haiman, Z. 2002, ApJ, 569, 558

Omukai, K. 2000, ApJ, 534, 809

Omukai, K., \& Palla, F. 2001, ApJ, 561, L55 
Palla, F. 2002, in Physics of Star Formation in Galaxies, ed. A. Maeder \& G. Meynet (Berlin: Springer), 16

Peebles, P.J.E. 1993, Principles of Physical Cosmology (Princeton: Princeton Univ. Press), 123

Qian, Y.-Z., \& Wasserburg, G.J. 2002, ApJ, 567, 515

Qian, Y.-Z., Sargent, W.L.W.,\& Wasserburg, G.J. 2002, ApJ, 569, L61

Reid, M.J. 1993, ARA\&A, 31, 345

Ricotti, M., Gnedin, N. Y., \& Shull, J. M. 2001, ApJ, 560, 580

Ripamonti, E., Haardt, F., Ferrara, A., \& Colpi, M. 2002, MNRAS, 334, 401

Sadat, R., Blanchard, A., Guiderdoni, B., \& Silk, J. 1998, A\&A, 331, L69

Santos, M. R., Bromm, V., \& Kamionkowski, M. 2002, MNRAS, 336, 1082

Schneider, R., Ferrara, A., Natarajan, P., \& Omukai, K. 2002, ApJ, 571, 30

Shapiro, P.R., \& Kang, H. 1987, ApJ, 318, 32

Siess, L., Livio, M., \& Lattanzio, J. 2002 ApJ, 570, 329

Silk, J. 1977, ApJ, 211, 638

Songaila, A. 2001, ApJ, 561, L153

Springel, V., \& Hernquist, L. 2002, MNRAS, in press (astro-ph/0206395)

Tegmark, M., Silk, J., Rees, M.J., Blanchard, A., Abel, T., \& Palla, F. 1997, ApJ, 474, 1

Thacker, R. J., Scannapieco, E., \& Davis, M. 2002, ApJ, in press (astro-ph/0205054)

Totani, T. 1997, ApJ, 486, L71

Tumlinson, J., \& Shull, J.M. 2000, ApJ, 528, L65

Umeda, H., \& Nomoto, K. 2002, ApJ, 565, 385

Vanhala, H.A.T., \& Cameron A.G.W. 1998, ApJ, 508, 291

Wijers, R.A.M.J., Bloom, J.S., Bagla, J.S., \& Natarajan, P. 1998, MNRAS, 294, L13 
White, S.D.M., \& Springel, V. 2000, in The First Stars, ed. A. Weiss, T. Abel, \& V. Hill (Berlin: Springer), 327

Yamada, M., \& Nishi, R. 1998, ApJ, 505, 148

This preprint was prepared with the AAS LATEX macros v5.0. 
Table 1. Stellar Populations at High Redshifts

\begin{tabular}{lcccc}
\hline \hline & $M_{*} / \mathrm{M}_{\odot}$ & $Z / \mathrm{Z}_{\odot}$ & $\mathrm{QW} \mathrm{SNe}^{\mathrm{a}}$ & Epoch \\
\hline Pop III..... & $\gtrsim 100$ & $<Z_{\text {crit }}$ & $\mathrm{VMS}$ & 1,2 \\
Pop II.5.... & $\gtrsim 10$ & $<Z_{\text {crit }}$ & $\mathrm{SN} \mathrm{II}(L)$ & 2 \\
Pop II....... & $\gtrsim 1$ & $>Z_{\text {crit }}$ & $\mathrm{SN} \mathrm{II}(H),(L)$ & 2,3 \\
\hline
\end{tabular}

aTentative identification with the different kinds of SNe proposed by Qian \& Wasserburg (2002). 